\title{
RECURRENT NASAL POLYP AND PANSINUS MUCOPYOCELE ASSOCIATED WITH BILATERAL BLINDNESS: A CASE REPORT
}

\author{
J. A. FASUNLA ${ }^{1}$, A. O. ADELEYE ${ }^{2}$, P. A. ONAKOYA ${ }^{1}$, C. A. OKOLO ${ }^{3}$ and G. O. NWAORGU ${ }^{1}$ \\ ${ }^{1}$ Department of Otorhinolaryngology, University College Hospital, Queen Elizabeth Road, PMB 5116, \\ Ibadan, Oyo State, Nigeria. ${ }^{2}$ Department of Neurosurgery, University College Hospital, Ibadan, Oyo State, \\ Nigeria ${ }^{3}$ Department of Pathology, University College Hospital, Ibadan, Oyo State, Nigeria.
}

Corresponding Author: Dr. James A. Fasunla

Conflict of Interest: None declared

\section{SUMMARY}

A 39 year old female with 24-month history of recurrent mucoid rhinorhea associated with progressive bilateral nasal blockage, headache, anosmia and bilateral impaired vision is reported. There was a past history of sinus surgery. The neuro-ophthalmic examination revealed left non-axial proptosis and visual acuity of nil light perception in both eyes at 3 meters. CT scan of the paranasal sinuses and brain showed markedly expanded left frontal sinus with sclerotic walls and expanded mixed density lesion in all the sinuses. She had surgery via trans-nasal, external and skull base approaches for effective clearance of the polypoidal masses and drainage of the sinus mucopyocele. Histological examination confirmed chronic inflammatory polyp. She remains stable to date although visual impairment and anosmia remained persistent. Patients with nasal polyposis require frequent follow-up even after surgical treatment for the early detection and management of complications to avoid consequences such as visual loss.

Keywords: Anosmia, Blindness, Mucocele, Paranasal sinuses, Pyocele

\section{INTRODUCTION}

Mucocele of the paranasal sinus is a chronic, expanding, mucosa-lined lesion characterized by mucous retention. ${ }^{1}$ The accumulation of mucous secretion and eventual secondary bacterial infection may result in the formation of a pyocele. ${ }^{1,2}$ This clinical condition has a tendency to distend and erode the sinus bony walls resulting, when the orbit is involved, in severe neuro-ophthalmic complications. ${ }^{3}$

Frontal and anterior ethmoidal sinuses are the commonest sites for mucocele or mucopyocele while the posterior ethmoidal, sphenoidal and maxillary sinuses are rarely involved. ${ }^{2,4-6}$ Multiple paranasal sinuses may be involved but bilateral pansinus mucopyocele is rare. ${ }^{6}$ Nasal polyps, infections, tumors
E-mail:ayofasunla@yahoo.com

(especially frontal osteoma), previous facial trauma and intranasal surgeries causing ostia blockage have been reported as notable causes of mucocele. ${ }^{7,8}$

This mucocele may become infected and become a pyocele. The infection stimulates production of cytokines [interleukin 1 (IL-1) and tumor necrosis factor (TNF)] from lymphocytes and monocytes, and increases synthesis of prostaglandin $\left(\mathrm{PGE}_{2}\right)$ and collagenase from fibroblast of the mucus lining. ${ }^{9}$

Lund and Milroy et al demonstrated and reported a high quantity of cytokines (IL-1, IL-6, and TNF), $\mathrm{PGE}_{2}$ and collagenase between the covering of the mucocele and sinus wall. ${ }^{9-11}$ This is responsible for the resorption and destruction of the sinus wall, allowing for the expansion of the mucocele.

Various surgical methods for successful management of this case involve external and trans-nasalendoscopic approaches. ${ }^{7,8}$ This communication is to highlight the interdisciplinary surgical management of what may be the first reported case of bilateral pansinus mucopyocele with bilateral blindness in an adult female in Africa.

Case report

A 39-year-old lady presented in our Ear, Nose and Throat (ENT) clinic with a 24-month history of progressive bilateral nasal blockage and nasal mass. There was associated recurrent mucoid nasal discharge, hyponasal speech and loss of perception of smell. There was no epistaxis, neck swelling, cheek or dental symptoms. Two months prior to presentation, she developed progressive bilateral visual impairment, worse in the left eye. There was associated headache but no neck pain, vomiting, or neurologic deficits. She had undergone the surgical procedure, 8 years previously, of bilateral external fronto-ethmoidectomy, nasal clearance and bilateral intranasal inferior meatal antrostomy for bilateral multi-sinusitis with nasal polyposis. Histology report confirmed chronic inflammatory polyp. 
Examination revealed a young woman with hyponasal speech and a slight degree of mouth breathing. She was not febrile and had no significant peripheral lymph node enlargement. There was telecanthus and bilateral healed Lynch-Howarth incision scars. Nasal examination revealed inflammed multiple polypoidal mass lesions covered by mucopurulent secretions protruding from and completely obliterating the right nasal cavity.

The mass was firm, sensitive to touch but did not bleed on contact and appeared to arise from the right lateral nasal wall. The left nasal cavity also had a pale polypoidal mass covered with mucopurulent secretions. The mass was non-sensitive, had no contact bleeding and appeared to arise from the lateral wall of left nasal. Examination of the throat revealed a granular posterior pharyngeal wall, and postnasal mucoid discharge.

Otoscopy revealed intact but dull tympanic membrane bilaterally. The neck appeared normal. The neuroophthalmic examination revealed left non-axial proptosis and visual acuity of nil light perception in both eyes at 3 meters. A clinical diagnosis of chronic rhinosinusitis with nasal polyposis was made.

The computed tomographic (CT) scan of the paranasal sinuses and brain revealed extensive mixed density lesions within all the paranasal sinuses. There was gross expansion of the left frontal sinus whose markedly sclerotic posterior wall caused marked compression of the left frontal lobe of the brain. Similar lesions filled both nasal cavities (Figures 1).
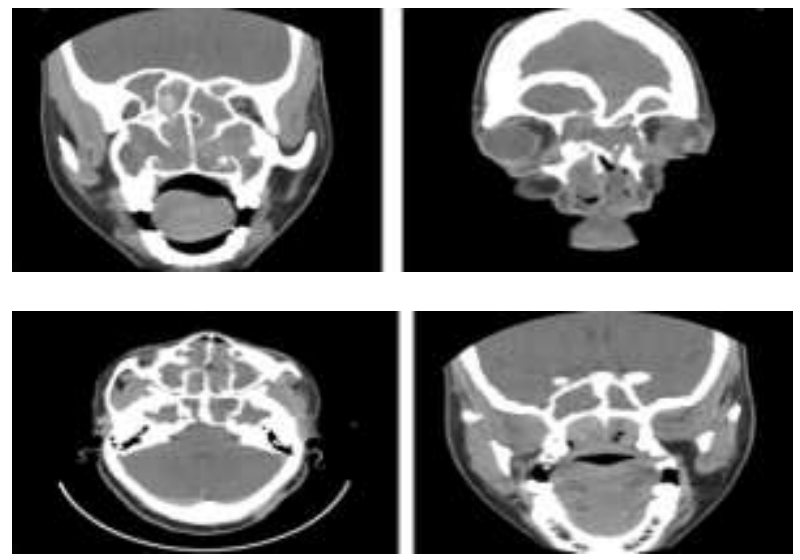

Figure 1:

Axial and coronal slides of computed tomographic scan of the paranasal sinuses and brain of the patient showing the markedly expanded left frontal sinus with sclerotic walls and expanded mixed density lesion in all the sinuses.

Using the Lund-Mackay grading system, a total numerical score of 24 was obtained. ${ }^{12}$ The diagnosis of chronic bilateral pansinusitis with recurrent nasal polyposis complicated by pansinus pyomucocele and bilateral blindness was made. An interdisciplinary strategy was then planned for the surgical excision of these extensive craniofacial lesions by a team of ENT surgeons and a skull base neurosurgeon.

The surgical procedure was carried out in one sitting and included bilateral nasal polypectomy, bilateral intranasal antrostomy, and bilateral fronto-ethmoidosphenoidectomy via bilateral fronto-nasal-orbital craniotomy. Reconstruction of Cranio-facial bone defects was facilitated using a titanium skull clamp, the CraniovFix $^{\mathrm{R}}$ (B Braun, Aesculap, Germany), Figure 2.

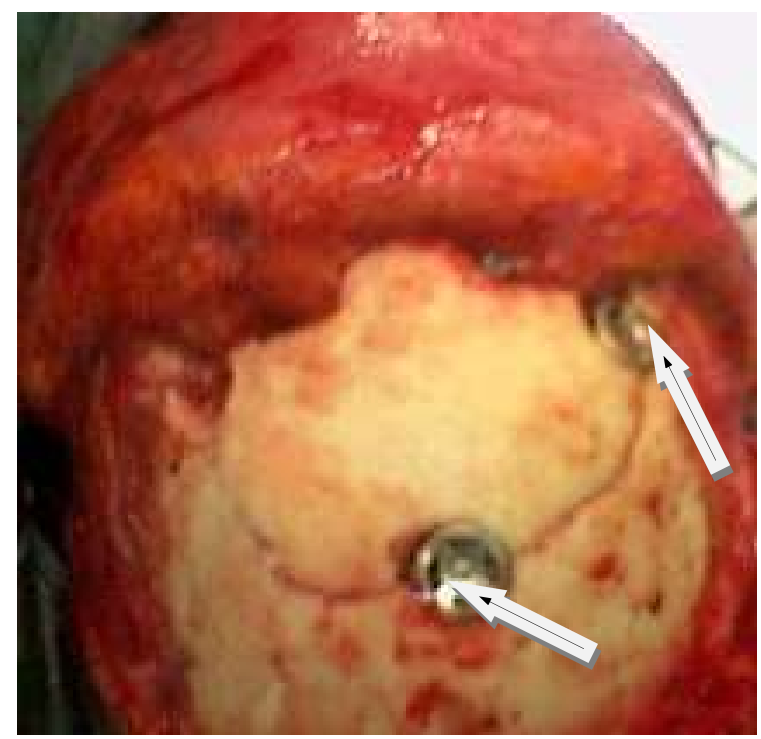

Figure 2: The craniofacial bone flap reconstruction after the bilateral frontal-nasal-orbital craniotomy; greatly facilitated by the titanium clamp (CranioFix, arrow)

The operative findings were bilateral polypoidal intranasal masses, bilateral defects in the supero-medial walls of the orbits communicating with the ethmoidal sinuses directly, complex mucopyocele in all the paranasal sinuses (Frontal, Ethmoidal, Sphenoidal, and maxillary sinuses) and sclerotic expansion of their walls. The histology of the specimen confirmed chronic inflammatory polyp

Postoperatively, she developed cerebrospinal fluid (CSF) rhinorrhea on the fourth postoperative day and this was successfully managed with serial lumbar CSF drainage. The persistent postoperative problems were bilateral blindness and anosmia.

\section{DISCUSSION}

This case report presents some noteworthy points of importance in clinical practice in a developing country. The first is the high price of a preventable bilateral 
blindness that this patient continues to pay for her delay in presentation for treatment of an otherwise benign treatable lesion. Another point is the beauty, and hence the imperative of interdisciplinary efforts between skull base neurosurgeons and ENT surgeons where neurological surgery and otorhinolaryngology meet in cases like this.

Mucoceles originate from obstructions to sinus drainage and can imitate malignant tumor radiologically. All paranasal sinuses can develop mucoceles but the frontal and anterior ethmoidal sinuses are the most commonly affected, followed by the sphenoid and then the maxillary sinus. ${ }^{5}$ This is because of the narrow and tortuous nature of the fronto-nasal duct that makes it more susceptible to stenosis or blockage by both extrinsic and intrinsic factors. These include infection, allergy, trauma, and surgical intervention. ${ }^{9}$

The presentation of paranasal sinus mucocele is usually unilateral; bilaterality is rare indeed. Our patient represents one of such rare occurrences of bilaterality. This might have occurred due to the associated recurrent bilateral nasal polyposis or the previous bilateral intranasal surgery which this patient had.

The old conventional intranasal surgical approach which does not employ the use of the current modern advanced surgical tools like nasal endoscopes to aid visualization of intranasal structures, has the great potential of inadvertently damaging or removing the thin bone of the orbit (lamina papyracea) and optic nerve with a possibility of damage to these structures. $^{13,14}$

This may then result in visual impairment. Although, the use of nasal endoscopes was not obtainable in the sub-region when this patient had her surgery 8 years ago, we expect that the blindness would have developed earlier if it was due to complications from that surgical procedure. This patient had preserved visual function until two months before she represented to us.

Mucus plugs of the paranasal sinus ostia are common but they do not usually result in mucocele. They cause only temporary ostia obstruction and are easily dislodged with medical treatment, with only the consequence of mucous retention which does not progress to mucocele. Mucoceles are relatively uncommon apparently because obstruction of the sinus drainage usually only leads to mucus retention within the sinus with only a small number progressing to mucocele. ${ }^{9}$
Development of the mucocele is noted to depend on the degree and duration of obstruction. ${ }^{9}$ Expansion may take place over many years or occur rapidly when there is superimposed infection that progresses to pyocele. In this report, the patient presented with features of complicated chronic rhinosinusitis with an 8 -year past history of bilateral sinonasal surgery.

This is a long enough time for the previously created fronto-nasal ducts to have been blocked again by polyps or stenosis. This patient had had 24 months of bilateral, progressive nasal blockage and the associated nasal polyps might have aided the blockage of the paranasal sinuses ostia resulting in mucous retention.

The development of bilateral visual loss, which is an unusual complication of mucocele may be because of the involvement of the ethmoidal sinuses which were hyperpneumatised and as such could have enclosed the optic nerve within its diseased cavities as seen in Onodi cell. $^{9}$

Mucocele of the fronto-ethmoidal region could cause pressure effect on the orbit thereby resulting in proptosis and impairment of vision. ${ }^{9}$ In addition, the sphenoid sinus is very close to the optic chiasma hence, its mucocele could also cause bilateral blindness ${ }^{4,9}$ as seen in this case.

Following the initial nasal surgery, the patient was lost to follow-up due to financial and other socio-economic constraints in our country. Hence, the routine intranasal examinations which could have led to early identification and treatment / curtailment of the recurrent nasal polyposis could not be achieved.

This patient only represented when she had already developed bilateral blindness. Early presentation of this patient would have prompted an imaging investigation which would have identified this lesion and institution of an early treatment intervention would have prevented this avoidable complication.

The current gold standard of imaging the paranasal sinuses is the CT scan which is very good at identifying any sinus mucocele and any other pathological changes within the paranasal sinus cavities. $^{9,11}$ Mucocele is demonstrated as a homogenous smooth walled mass, expanding the sinus, with thinning or loss of the cortical bone altogether. There may be evidence of new bone formation or sclerosis (Figure 1), as the mucopyocele may initiate a process of bone resorption and expansion. ${ }^{11}$

Successful extirpation of such cases therefore should include open transcranial extradural approach as 
employed in this case. Unfortunately, visual recovery is hardly ever achieved when the visual loss is severe and longstanding as in this case. ${ }^{4,15}$

In conclusion, a patient with nasal polyposis requires frequent follow-up even after surgical treatment. This way, any recurrence can be identified early and managed appropriately in order to prevent such unsalvageable complications as bilateral blindness.

\section{REFERENCES}

1. Menendez-Colino LM, Bernal SM, Benitez SP, Alobid I, Guillemany-Toste JM: Technical aspects in endoscopic surgery of frontal mucopyocele. Acta Otorrinolaringol Esp 2003;54:98-101.

2. Cinar U, Yigit O, Uslu B, Alkan S: Pyocele of the middle turbinate: a case report. Kulak Burun Bogaz Ihtis Derg 2004;12:35-38.

3. Chen TM, Lee TJ, Huang TS: Endoscopic sinus surgery for the treatment of frontoethmoidal mucocele complicated with orbital abscess: a case report. Changgeng Yi Xue Za Zhi 1997;20:39-43.

4. Selvapandian S, Rajshekhar V, Chandy MJ: Mucoceles: a neurosurgical perspective. Br J Neurosurg 1994;8:57-61.

5. Arnaud B, Zaghloul K, Dupeyron G, Malrieu, C: Mucocele of ophthalmologic expression and treatment apropos of 46 cases. Bull Soc Ophthalmol Fr 1989;89:1221-1224.

6. Pinto JA, Cintra PP, de Marqui AC, Perfeito DJ, Ferreira RD, da Silva RH: Mucopyocele of the middle turbinate: a case report. Braz J Otorhinolaryngol 2005;71:378-381.

7. Rubin JS, Lund VJ, Salmon B: Frontoethmoidectomy in the treatment of mucoceles. A neglected operation. Arch Otolaryngol Head Neck Surg 1986;112:434-436.

8. Natvig K, Larsen TE: Mucocele of the paranasal sinuses. J Laryngol Otol 1978;92:1075-1082.

9. Lund VJ: The orbit in MacKay LS, Bull TR (eds.): Rhinology: Scott-Brown's Otolaryngology, ed 2, Great Britain: Butterworth-Heinemann 1997; vol 4, pp 1-19.

10. Lund VJ, Milroy CM: Frontoethmoidal mucoceles: a histopathological analysis. $J$ Laryngol Otol 1991;105:921-923.

11. Dolan KD: Paranasal sinus radiology, Part 3B: Sphenoidal sinus. Head Neck Surg 1983;5:237250.

12. Lund VJ, Mackay IS. Staging in rhinosinusitis. Rhinology 1993;31:183-184.

13. Maniglia AJ. Fatal and major complications secondary to nasal and sinus surgery. Laryngoscope 1989;99: 276-283.

14. Stammberger H. Functional endoscopic sinus surgery: the Messerklinger technique. Philadelphia: Decker 1991; pp283,

15. Darouassi Y, Righini CA, Reyt E: Mucoceles of the sphenoidal sinus: a report of four cases and review of the literature. $B$-Ent 2005;1:181-185. 